\title{
Presente y futuro de las publicaciones médicas
}

\section{Present and future of medical journals}

\author{
Borja Ibáñez ${ }^{a, b, c, *}$ y Josep Rodés-Cabau ${ }^{d}$ \\ a Centro Nacional de Investigaciones Cardiovasculares (CNIC), Madrid, España \\ b Instituto de Investigación Sanitaria de la Fundación Jiménez Díaz, Madrid, España \\ ${ }^{\mathrm{c}}$ CIBER de Enfermedades Cardiovasculares (CIBERCV), Madrid, España \\ d Department of Cardiology, Quebec Heart and Lung Institute, Laval University, Quebec City, Quebec, Canadá
}

El acceso a la literatura médica ha sufrido un cambio radical en los últimos años. En menos de dos décadas se ha pasado de un sistema basado en papel a un sistema de envío digital en red. El desarrollo de la informática, y sobre todo de internet, ha revolucionado no solo el formato de envío de manuscritos, sino también la rapidez con que se preparan estos (incluyendo gestores informáticos de referencias adaptables al formato diferente de cada revista). Esta revolución también ha afectado a la velocidad de acceso a la información. Las bases de datos que se generaban a principios del siglo XXI pueden considerarse "prehistóricas» comparadas con las existentes hoy. La digitalización de las historias clínicas y la creación de cuadernos de recogida de datos informáticos aceleran de manera exponencial la preparación y el análisis de los datos incluidos en las investigaciones. Incluso la formación de los investigadores es en la actualidad mucho más transversal, y es frecuente encontrar en los equipos de investigación expertos en estadística que facilitan el análisis de los datos. Sin embargo, todos estos cambios tan importantes se quedan pequeños en comparación con el acceso existente a la información médica en general. Pese a que el acceso a la literatura no es libre en general (tema comentado más adelante), sí existe un acceso muy alto a una gran cantidad de información. Todo ello ha resultado en un incremento exponencial de la cantidad de artículos que las revistas reciben mensualmente. Además, esto se ve magnificado por la productividad creciente de países emergentes o potencias, como es el caso de China, que ha multiplicado notablemente el número de artículos científicos en los últimos años. A modo de ejemplo ilustrativo, una de las revistas de referencia en el campo de las enfermedades cardiovasculares, Journal of the American College of Cardiology (JACC), recibió de media unos 4.000 manuscritos anuales (3.200 artículos originales/ revisiones) en un periodo de 5 años desde que el español Valentín Fuster fue nombrado su editor jefe en 2014. Debido a la aceleración en la generación de conocimiento y la tecnificación de diferentes subespecialidades, la audiencia de las revistas de cardiología tiene particular interés en algunos campos concretos. El gran volumen y la progresiva especialización de los manuscritos enviados a las revistas, junto con el número limitado de publicaciones mensuales de las mismas, resultan en una tasa de éxito reducida, pues se rechazan artículos de calidad alta e interés para la comunidad cardiológica. Siguiendo con el ejemplo del JACC, la tasa de aceptación de artículos originales/revisiones en el periodo citado fue solo de aproximadamente el 9\%. Estas circunstancias han resultado en la creación de las revistas «hermanas» (sister journals en inglés) de las grandes revistas, como son las especializadas en intervencio- nismo, imagen, insuficiencia cardiaca o arritmias, entre otras. Debido a que es esperable que este incremento del número de manuscritos remitidos a las revistas siga en ascenso, también el número de revistas de cardiología y de sus subespecialidades se anticipa que seguirá aumentando. Lejos de diluir la relevancia de las revistas, este crecimiento enriquece la ciencia médica e incrementa el acceso al conocimiento y la difusión de este. Una práctica frecuente en las revistas de alto impacto para los artículos de gran calidad que son considerados muy especializados es ofrecer a los autores la transferencia del manuscrito a una revista «hermana». Siguiendo con el ejemplo del JACC, en los años mencionados, en torno a un $4 \%$ de los artículos originales/revisiones recibidos fueron referidos y finalmente aceptados por la revista JACC: Cardiovascular Interventions. Esta práctica acelera el proceso de publicación y orienta a los autores sobre un posible interés de la revista en cuestión.

Resulta paradójico que, con toda la digitalización descrita previamente, las principales revistas científicas, las de cardiología en particular, sigan teniendo un formato clásico de revista impresa con un número limitado de artículos publicados por año. La tendencia global hace pensar que este formato no perdurará mucho tiempo. Con esta idea nacieron las revistas solo en formato digital, muchas de ellas de acceso libre (open access journals). Pese a su atractivo, su impacto no se acerca al de las revistas clásicas, lo que nos hace abrir una reflexión sobre qué es lo que buscan los lectores y los autores de manuscritos en las revistas. De modo general el lector quiere tener acceso a información que le ilustre con avances de actualidad y que le resuma de manera sencilla la evidencia existente sobre un tema en particular. En muchas ocasiones, el lector no es capaz de evaluar si las investigaciones publicadas se han realizado de manera correcta, o si se habrá revisado apropiadamente la literatura existente sobre el tema. Es por ello que busca revistas «de cabecera» que tengan un sello de calidad que garantice que lo publicado ha pasado todos los filtros y por tanto es veraz, bien realizado y equilibrado en su exposición. En este sentido, la figura de los editores es de importancia capital, ya que en cierto modo imprimen un carácter particular a la revista. Si bien existen diferentes sellos de calidad para evaluar las revistas, el factor de impacto (FI) es el más extendido para tabular el nivel de una revista en su entorno. El FI, un cálculo «oficial» que se realiza cada año, es el resultado del cociente entre las citas recibidas durante un año a artículos publicados en una revista en los dos años previos y el número de artículos publicados en el mismo periodo. A mayor FI, mayor calidad se presupone a la revista. El FI lo

\footnotetext{
* Autor para correspondencia: Departamento de Investigación Clínica, Centro Nacional de Investigaciones Cardiovasculares (CNIC), Melchor Fernández Almagro 3, 28029 Madrid, España.

Correo electrónico: bibanez@cnic.es (B. Ibáñez).
} 
calcula anualmente una empresa privada (actualmente Clarivate Analytics) y establece el ranking de las revistas dentro de su campo. Existen otras métricas que evalúan el impacto de las revistas (Google Académico ${ }^{1}$ está ganando mucho terreno), pero al igual que el FI son imperfectas y no evalúan todos los aspectos de calidad de una revista. Como detallaba el propio editor jefe del JACC en un editorial de $2017^{2}$, el FI es una métrica curiosa y caprichosa, ya que la presencia de un artículo muy citado (las guías de práctica clínica como ejemplo más paradigmáticol tiene un impacto muy importante sobre el FI global de la revista, incluso aunque el resto de los artículos publicados no reciban ninguna atención. Por otro lado, el autor de un trabajo busca publicar en una revista que vaya a dar la mayor visibilidad posible a su investigación, y que le aporte prestigio profesional. De nuevo, el FI desempeña un papel muy importante al elegir a qué revista enviar un trabajo para su valoración. Además, los científicos deben de estar continuamente solicitando financiación (nacional e internacional) para sus investigaciones, y la mayoría de las agencias de evaluación miden los currículos en función del número y la calidad de las publicaciones, que suelen baremarse por su FI y su posición relativa comparada con otras revistas de su campo. Por tanto, se genera un círculo vicioso en el cual los lectores y los autores apuntan a las mismas revistas, y son estas las que pueden elegir de primera mano los trabajos de mayor relevancia y ello les garantiza mantener un buen nivel de citaciones, un alto FI y, por tanto, seguir siendo atractivas para todo el público. Aunque existen muchas voces en contra de este sistema establecido, son tantos los factores que giran alrededor de las métricas que es difícil imaginar un futuro donde los lectores y los autores abandonen estos parámetros.

Un aspecto muy relevante al actuar como comité editorial de una revista científica es tener claro cuál es la audiencia a la que va a dirigirse $y$, sobre todo, qué tipo de información quiere transmitirse. En relación al segundo punto, las revistas pueden tener una vocación puramente científica (con predominio de artículos originales) o también educativa (con predominio de revisiones de temas de alto interés clínico). El formato mixto es el que con más frecuencia eligen las revistas. Cabe destacar que algunas revistas con un enfoque exclusivamente educativo han llegado a tener un FI muy alto en el campo de la cardiología, como por ejemplo Nature Reviews Cardiology, que en 2017 se posicionó en los primeros lugares del FI de su campo.

En los últimos años, la revolución de internet ha resultado en la creación y el crecimiento vertical de las redes sociales, que se están convirtiendo en un ingrediente muy importante de diseminación de los artículos publicados en las revistas ${ }^{3}$. Las redes sociales son cruciales para diseminar la investigación no solo entre los propios científicos/médicos, sino también entre el público general, con lo cual se contribuye a promocionar la salud. Este aspecto de difusión a la audiencia no médica es crítico y cada vez va a tener mayor relevancia. Existen métricas de reciente creación que cuantifican el impacto de las publicaciones por su actividad en las redes sociales, como es Altmetric score ${ }^{4}$. En un estudio en el que se evaluaron los Altmetric scores de cuatro de las revistas más relevantes en el campo de la cardiología se hallaron algunos datos interesantes ${ }^{5}$ : a) los Altmetric scores de las revistas de cardiología son en general muy altos; b) más de la mitad de los artículos con mayor diseminación no fueron publicaciones originales, sino editoriales, puntos de vista, guías de práctica clínica y documentos de consenso; c) los artículos con un impacto más alto acorde con esta métrica fueron los del campo de la nutrición y estilos de vida; y d) los artículos open access no tuvieron más impacto que los de pago por lectura (pay per read en inglés) $)^{5}$.
Un último tema muy relevante, y a la vez controvertido, es el coste asociado a la publicación de un artículo y el acceso a este. Muchas revistas (ciertamente las de mayor FI) suelen tener contratos en exclusividad con grandes empresas editoriales que se encargan de formatear, publicar y distribuir los artículos. Para tener acceso a los artículos completos, las universidades, las instituciones de investigación e incluso los profesionales particulares pagan una suscripción. Este pago por lectura ciertamente limita la difusión del conocimiento. Muchos autores deciden pagar una cuota cuando su artículo es aceptado por una revista para que sea de libre lectura (open access) y cualquier persona pueda acceder a él sin necesidad de suscripción. Esta cuota (habitualmente entre 2.500 y $4.500 €$ ) es una partida contemplada en los fondos públicos concedidos a los autores. Las revistas envían los manuscritos a revisores externos que trabajan sin remuneración, por altruismo y responsabilidad. Por tanto, nos encontramos ante una situación compleja en la que el creador de la obra (autor), el evaluador (revisores externos y en muchas ocasiones comités editoriales) y el consumidor final (lector) pagan por ella, y los beneficios económicos son solo para el distribuidor de la mercancía (empresa editorial) que no ha intervenido en el proceso más que para su maquetación y distribución. El hecho de que la investigación realizada con fondos públicos no sea de acceso libre, y que por tanto no sea aprovechada por toda la comunidad y el público, es muy cuestionable. Por ello, en algunos países europeos (Suecia el más reciente) se ha decidido cancelar las suscripciones con grandes empresas editoriales como empuje hacia un acceso libre a la ciencia ${ }^{6}$.

En resumen, el presente de las publicaciones médicas está sufriendo un cambio radical, principalmente guiado por el desarrollo de internet, la informática y los medios de comunicación sociales. El destino de este camino es difícil de intuir, pero parece que las revistas pasarán a ser por completo digitales y de acceso (cuasi) libre para los lectores, con unos sellos de calidad diferentes del FI clásico. Debido a la grandísima cantidad de información disponible, la creación de revistas subespecializadas es una tendencia que irá en aumento y facilitará a los profesionales estar actualizados en su campo concreto de trabajo. REC: Interventional Cardiology cumple muchos de estos ingredientes futuristas $y$, por tanto, podemos augurarle una exitosa andadura de la mano de un excelente comité editorial y bajo el sello de calidad de Revista Española de Cardiología.

\section{CONFLICTO DE INTERESES}

Los autores declaran no tener ningún conflicto de intereses.

\section{BIBLIOGRAFÍA}

1. Google Scholar. Disponible en: https://scholar.google.com. Consultado 28 Ene 2019.

2. Fuster V. Impact Factor: A Curious and Capricious Metric. J Am Coll Cardiol. 2017;70:1530-1531

3. Walsh MN. Social Media and Cardiology. J Am Coll Cardiol. 2018;71:10441047 .

4. Almetric Attention Score. Disponible en: https://www.altmetric.com/ about-our-data/the-donut-and-score/. Consultado 28 Ene 2019.

5. Patel RB, Vaduganathan M, Bhatt DL, Bonow RO. Characterizing High-Performing Articles by Altmetric Score in Major Cardiovascular Journals. JAMA Cardiol. 2018. https://doi.org/10.1001/jamacardio.2018.3823.

6. Else H. Europe's open-access drive escalates as university stand-offs spread. Nature. 2018;557:479-480 